\title{
Pathogenesis of diabetic kidney disease
}

Received 08 January 2021

Accepted 14 February 202

Sydney C.W. Tang*, Anthony T.P. Chan, Wai Han Yiu, Kar Neng Lai

Division of Nephrology, Department of Medicine, The University of Hong Kong, Queen Mary Hospital, Hong Kong, China

Abstract

Diabetic kidney disease (DKD) is characterized by an accumulation of extracellular matrix proteins such as collagen and fibronectin in the kidney, resulting in tubulointerstitial fibrosis, glomerular mesangial hypertrophy and expansion, thickening of the glomerular basement membrane, podocyte foot process effacement, and inflammation due to the infiltration of monocytes and macrophages. All of these factors contribute to kidney function loss and can ultimately lead to progressive chronic kidney disease and kidney failure. In the review, we summarize the current state of knowledge in the pathogenesis of diabetic kidney disease to include the impact of genetic and environmental factors, hemodynamic changes, glycemic control, inflammation, proteinuria and novel mechanisms such as non-coding RNAs and lipotoxicity.

Keywords

diabetic kidney disease $\bullet$ genetics $\bullet$ hemodynamics $\bullet$ proteinuria $\bullet$ inflammation $\bullet$ lipotoxicity $\bullet$ non-coding RNAs

\section{Introduction}

The development and progression of diabetic kidney disease (DKD), a highly prevalent complication of diabetes mellitus, is influenced by both genetic and environmental factors. On top of this, many other pathways interact to induce DKD which is an important contributor to the morbidity of patients with diabetes mellitus. As the incidence of DKD as the primary cause of treated kidney replacement therapy increases across the world, there is a clear need for an improved understanding of disease pathogenesis to inform the development of more efficacious treatment strategies. Here, we summarize what is currently known in the initiation and progression DKD.

\section{Genetic and Environmental Factors}

Although the risk of development of DKD is equal in both type 1 and type 2 diabetes, only $30-40 \%$ of patients with type 1 or type 2 diabetes will ultimately develop nephropathy. Although barriers to care are likely to account for some of these interpopulation differences, polygenetic factors may also contribute.

Familial clustering of DKD has been reported in type 1 and type 2 diabetes in Caucasian and non-Caucasian populations. In a type 1 diabetic who has a first-degree relative with diabetes and nephropathy, the risk of development of DKD is at $83 \%$. However, the frequency is only $17 \%$ if there is a first-degree relative with diabetes but without nephropathy [1]. In type 2 diabetes, familial clustering has been well documented in Pima Indians [2]. A familial determinant is also suggested by higher albumin excretion rates in the offspring of type 2 diabetic patients with nephropathy. The risk is particularly high in the offspring if the mother had been hyperglycemic during pregnancy, perhaps because this causes a reduced formation of nephrons ("nephron underdosing") in the offspring [3, 4]. Low birth weight and nephron underdosing are also associated with hypertension, metabolic syndrome, and perhaps DKD, although for DKD the data are somewhat controversial. Nephron underdosing [5] is believed to lead to compensatory glomerular hypertrophy and increased single-nephron glomerular filtration rate (GFR), thus aggravating glomerular injury in diabetes.

In patients with type 1 diabetes the estimate of heritability for nephropathy was $35 \%$, although replication studies did not identify any single genetic variance reaching whole-genome levels of significance.

Environmental factors, especially diet, may also be involved in the pathogenesis of diabetes and diabetic nephropathy (DN). One of the strongest risk factors is the intake of soft drinks containing added sugars such as sucrose or high-fructose corn syrup. Fructose increases uric acid levels, a potent predictor for the development of type 2 diabetes as well as DN, probably via uric-acid-inducing oxidative stress and endothelial dysfunction.

\section{Hemodynamic Changes}

Hyperfiltration is common in early diabetes but can be corrected with good glycemic control. Increased GFR involves glucose-dependent effects that cause afferent arteriolar dilation, which is mediated by a range of vasoactive mediators, including insulin-like growth factor 1 (IGF-1), transforming growth factor $\beta$ (TGF- $\beta$ ), vascular endothelial growth factor 
(VEGF), nitric oxide (NO), prostaglandins, and glucagon. Over time, development of vascular disease of the afferent arteriole may result in permanent alterations in renal autoregulation that favor glomerular hypertension. Renal injury in DKD is caused not only by hemodynamic disturbances (e.g., hyperfiltration, hyperperfusion) but also by disturbed glucose homeostasis, and the two pathways interact.

DKD is also associated with tubular abnormalities: hyperfiltration increases the colloid osmotic pressure in postglomerular capillaries, facilitating reabsorption of sodium in the proximal tubule. Angiotensin II (Ang II) also appears to have a role, causing hypertrophic proximal tubular growth and increased sodium reabsorption [6]. Specific inhibition of the sodiumglucose co-transporter 2 (SGLT2) in proximal tubular cells is associated with reduced progression of diabetic kidney disease, emphasizing the role of tubuloglomerular feedback and glomerular hyperfiltration in DN [7].

\section{Renal Hypertrophy and Mesangial Matrix Expansion}

Renal growth occurs early after the onset of diabetes. Glomerular enlargement is associated with increased numbers of mesangial cells, mesangial cell hypertrophy, and increase of capillary loops, thus enhancing the filtration surface area. Renal tubular hypertrophy is primarily the result of both tubular epithelial cell proliferation and hypertrophy.

Experimentally, it has been ascertained that avoidance of hyperglycemia prevents renal hypertrophy. Hyperglycemia causes hypertrophy by stimulating growth factors in the kidney, including IGF-1, epidermal growth factor (EGF), platelet-derived growth factor (PDGF), VEGF, TGF- $\beta$, and Ang II. Hyperglycemia also induces the expression of thrombospondin, which is a potent activator of latent TGF- $\beta$. Experimentally, neutralizing antibodies to TGF- $\beta$ attenuated diabetes-related renal hypertrophy and extracellular matrix $(E C M)$ accumulation and preserved renal function.

\section{Inflammation}

Inflammatory processes and immune cells are involved in the development and progression of DN [8]. Glomerular and interstitial infiltration by monocytes/macrophages and activated $\mathrm{T}$ lymphocytes as well as heightened NIrp-3 inflammasome activation are observed both in human and experimental DN [9]. Chemokines and their receptors, in particular monocyte chemotactic protein 1 (MCP-1/CCL2), RANTES/CCL5, IL-6, TNF-receptors, as well as adhesion molecules (e.g., ICAM-1) seem to contribute to this [8, 10]. Soluble TNF-receptors appear to be a robust biomarker for progressive kidney disease in both type 1 and type 2 diabetes. Bardoxolone methyl, as an inducer of the KEAP1Nrf2 pathway, exhibits anti-inflammatory effects, but in a phase III clinical trial it caused significantly more adverse effects and increased mortality. A trial using the oral CCR2 inhibitor CCX140-B for 52 weeks yielded more promising results in reducing residual albuminuria in type 2 diabetic subjects [11].

\section{Mechanisms Underlying Proteinuria}

Widening of the glomerular basement membrane (GBM) is associated with accumulation of type IV collagen and a net reduction in negatively charged heparin sulfate proteoglycan. The expression of one permeability-controlling protein, nephrin, is abnormally low in DN [8]. The transcription of nephrin is suppressed by Ang II and restored by inhibitors of the renin-angiotensin system (RAS). In addition, in $\mathrm{DN}$, apoptosis of podocytes is triggered by various factors, including Ang II and TGF- $\beta$, and adhesion of podocytes to the GBM is reduced by AGE-induced suppression of neuropilin-1. Podocyte loss also follows hyperglycemia-induced reactive oxygen species (ROS) generation, resulting in podocyte-apoptosis or -detachment. Migration of podocytes is also attenuated by the reduction of neuropilin-1, thereby preventing surviving podocytes from covering denuded areas of GBM, which promotes development of focal segmental glomerulosclerosis (FSGS).

\section{Tubulopathic Changes}

Although glomerulosclerosis is a cardinal feature of DN, tubulointerstitial injury ultimately determines the rate of attrition of renal function. In vitro studies have demonstrated the pathogenic role of various diabetic substrates in promoting tubule hypertrophy, stimulating ECM production, and inducing a proinflammatory and profibrotic phenotype in proximal tubular epithelial cells (PTECs), including high glucose, accumulation of glycated proteins, AGEs and their carbonyl intermediates, elevated intrarenal Ang II, oxidative stress, and hypertension-induced mechanical stress [12]. Another mechanism by which glucose may promote diabetic tubulopathy is by conversion through the polyol pathway to fructose, where it is degraded by local fructokinase to induce oxidative stress and local inflammation.

Glomerular cells, tubular epithelial cells, macrophages/lymphocytes, and fibroblasts/myofibroblasts all contribute to matrix accumulation along the glomerular and tubular basement membranes and within the interstitial space. In particular, matrix-producing myofibroblasts promote progression of fibrosis in DKD by facilitating deposition of interstitial ECM.

Hypoxia in DKD is exacerbated by the progressive hyalinosis of the afferent and efferent arterioles and loss of peritubular capillaries. In experimental chronic renal injury, hypoxia is an important factor which aggravates interstitial fibrosis, partly by the induction of TGF- $\beta$ and VEGF. The transition of tubular epithelial cells into fibroblasts is stimulated by cellular hypoxia [13]. The induction of growth factors and cytokines is mediated by hypoxia-inducible factor 1 , which can be amplified by Ang II; whether early treatment of anemia with 
erythropoietin or HIF1 stabilizers delays DKD progression remains unproven.

\section{Role of Glucose Control}

The role of glucose is demonstrated by evidence that tight glycemic control retards the development of DKD [14-17].

The detrimental role of high circulating glucose leads to the conceptualization that high glucose can be considered as a metabolic danger signal that is effected via toll-like receptors (TLRs), a conserved family of pattern recognition receptors that play a fundamental role in innate immunity [18]. In particular, TLR4 is overexpressed in the renal tubules of human DN biopsies $[19,20]$. In vitro, silencing TLR4 ameliorated high-glucose-induced tubular cell inflammation. In experimental DN, either systemic deletion or the application of a TLR4 antagonist conferred renoprotection [20].
In addition, TLRs are also expressed in other resident renal cell types such as podocytes and mesangial cells as well as infiltrating macrophages that could act in concert to bring about an inflammatory phenotype observed in diabetic kidney disease (Figure 1). A key component of innate immunity includes the NADPH oxidase pathway, which is expressed primarily in the lysosomes of phagocytic cells, but also in the kidney. Enhanced Nox4 in podocytes may contribute to glomerular disease involving a pathway linking Nox to the citric acid cycle [21]

\section{Renin-Angiotensin-Aldosterone System}

In patients with DN, Ang II has many nonhemodynamic effects and mediates cell proliferation, hypertrophy, ECM expansion, and cytokine (TGF- $\beta$, VEGF) synthesis [22]. Therefore, ACE inhibitors and ARBs presumably act by hemodynamic as well as nonhemodynamic actions.

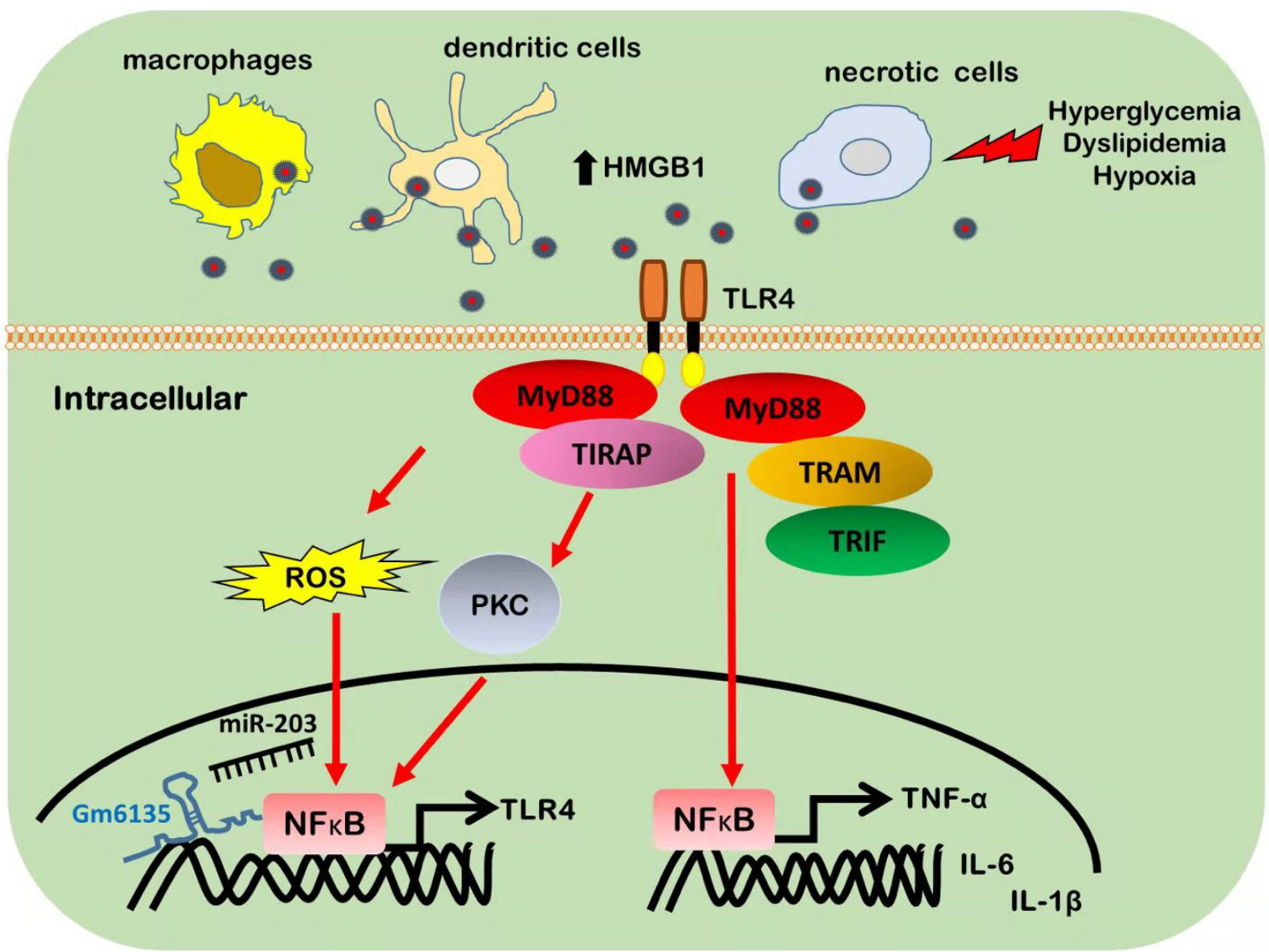

Figure 1. Activation of TLR4 signaling in DKD. In response to diabetic stimuli, such as hyperglycaemia, dyslipidaemia and hypoxia, dendritic cells, macrophages, and necrotic cells release HMGB1 into the extracellular fluid. The cell surface expression of TLR4 is also upregulated in response to high glucose levels. Binding of HMGB1 to TLR4 expressed on tubular epithelial cells promotes TLR4 dimerization, which triggers a downstream inflammatory cascade and the production of ROS. Recruitment of adapter proteins, including MyD88, TIRAP, TRIF, and TRAM, to the intracellular domain of TLR4 leads to translocation of NF-KB to the nucleus and the transcription of genes that encode proinflammatory cytokines, including IL-6, IL-1 $\beta$, and TNF. The transcription of TLR4 is also regulated by the TLR4-NF-KB pathway via recruitment of TIRAP, activation of PKC, and the generation of ROS. The long non-coding RNA Gm6135 upregulates and miR203 suppresses transcription of TLR4 via binding to the TLR4 promoter. HMGB1, high mobility group protein B1; MyD88, myeloid differentiation factor 88; ROS, reactive oxygen species; TIRAP, TIR-domain containing adaptor protein; TRIF; TIR-domain-containing adaptor inducing interferon; TLR, Toll-like receptor; TRAM, TRIF-related adaptor molecule. 
Aldosterone accelerates progression in renal damage models independently of Ang II. In DN, aldosterone escape has been linked to progression of proteinuria. Aldosterone synthesis is stimulated in $\mathrm{DN}$, and this steroid hormone stimulates the synthesis of other proinflammatory and profibrogenic cytokines (MCP-1, TGF- $\beta$ ) [23]. Fenerenone is a nonsteroidal aldosterone receptor antagonist that has shown promise in the treatment of DN [24].

Other vasoactive agents may also be involved in the pathogenesis of DN, including alterations in systemic or intrarenal production of endothelin, NO, the kallikrein-kinin system, and natriuretic peptides. A randomized controlled clinical trial examining the efficacy of a selective endothelin-A receptor antagonist showed promising results [25].

\section{Novel Mechanisms}

In recent years, there have been a plethora of studies on the potential pathogenesis of DN, such as non-coding RNA and lipotoxicity.

Increasing evidence shows epigenetic mechanisms, involving chromatin histone modifications, DNA methylation, and non-coding RNAs contribute to the development of DN. In the interest of space, we only discuss the role of ncRNAs here. The first miRNA shown to have a functional role in DKD was miR-192, which acts by targeting key repressors to promote the expression of ECM and collagen, and to augment the pro-fibrotic effects of TGF $\beta 1$. Numerous miRNAs are now thought to regulate key features of DKD, such as podocyte apoptosis, the accumulation of ECM, glomerular and tubular hypertrophy, and fibrosis. Detailed mechanisms on the role of ncRNAs and DKD have been reviewed by Kato and Natarajan [26].
LncRNAs are long transcripts ( $>200$ nucleotides and up to $\sim 100 \mathrm{~kb}$ in length) that have many similarities with mRNAs but lack protein-coding (translation) potential. Increasing evidence suggests that IncRNAs have distinct cellular roles that affect various biological mechanisms and processes, including gene transcription, splicing, mRNA stability, epigenetic regulation, cell cycle control, differentiation, and the immune response. Evidence suggests that a number of IncRNAs could play a pivotal role in development and progression of DKD either via direct involment or as indirect mediators of nephropathic pathways, such as TGF- $\beta 1$, NF-kB, STAT3, and GSK-3 $\beta$ signaling. Some IncRNAs may therefore become biomarkers for early diagnosis or prognosis of DKD or as therapeutic targets for retarding or even reversing the progression [27].

Lipotoxicity is another pathway that is increasingly implicated in DN. Obesity is a global epidemic resulting from an interplay between genetic and environmental factors such as sedentary lifestyle and unhealthy dietary habits characterized by a high consumption of food abundant in energy and fat. A myriad of metabolic disturbances including chronic systemic low-grade inflammation and insulin resistance are directly or indirectly associated with not only obesity, but also other metabolic diseases like metabolic syndrome, obesity-related type 2 diabetes mellitus, non-alcoholic fatty liver disease, and cardiovascular disease. Animal and in vtiro studies have identified saturated fatty acids the dominant non-esterified fatty acid in the circulation of obese subjects that act as non-microbial agonists to trigger the inflammatory response via activating TLR4 signaling. Recent advances in understanding the crosstalk between TLR4 and SFAs to induce inflammation and insulin resistance in multiple organs have been reviewed by Li et al. [28].

\section{Funding Sources}

This study was supported by grants from the Research Grants Council of Hong Kong (grant nos. 17108719, 17119818, and C7018-16G); and by philanthropic donations from Dr. Rita T Liu SBS of L \& T Charitable Foundation Ltd. \& Bingei Family of Indo Café, Mr. Winston Leung, Mr. K. K. Chan, Ms. Siu Suet Lau and the Divine Grace Foundation Ltd. W.H.Y. is supported by the Mr \& Mrs Tam Wing Fan Edmund Renal Research Fund and an Endowment Fund established at the University of Hong Kong for the Yu Professorship in Nephrology awarded to SCWT.

Conflict of Interest

Sydney C.W. Tang is an assiciate editor of the journal. There is no other conflict of interest declared.

\section{REFERENCES}

[1] Seaquist ER, Goetz FC, Rich S, Barbosa J. Familial clustering of diabetic kidney disease. Evidence for genetic susceptibility to diabetic nephropathy. N Engl J Med 1989; 320:1161-5. [2] Nelson RG, Knowler WC, Pettitt DJ, Saad MF, Bennett $\mathrm{PH}$. Diabetic kidney disease in Pima Indians. Diabetes Care 1993; 16:335-41.
[3] Nelson RG, Morgenstern H, Bennett PH. Intrauterine diabetes exposure and the risk of renal disease in diabetic Pima Indians. Diabetes 1998; 47:1489-93.

[4] Amri K, Freund N, Duong Van Huyen JP, Merlet-Benichou C, Lelievre-Pegorier M. Altered nephrogenesis due to maternal diabetes is associated with increased expression of 
IGF-II/mannose-6-phosphate receptor in the fetal kidney. Diabetes 2001; 50:1069-75.

[5] Zandi-Nejad K, Luyckx VA, Brenner BM. Adult hypertension and kidney disease: The role of fetal programming. Hypertension 2006; 47:502-8.

[6] Satriano J, Vallon V. Primary kidney growth and its consequences at the onset of diabetes mellitus. Amino Acids 2006; 31:1-9.

[7] Fitchett D, Zinman B, Wanner C, Lachin JM, Hantel S, Salsali $A$, et al. Heart failure outcomes with empagliflozin in patients with type 2 diabetes at high cardiovascular risk: Results of the EMPA-REG OUTCOME ${ }^{\circledR}$ trial. Eur Heart $\mathrm{J}$ 2016; 37:1526-34.

[8] Reidy K, Kang HM, Hostetter T, Susztak K. Molecular mechanisms of diabetic kidney disease. J Clin Invest 2014; 124:2333-40.

[9] Shahzad K, Bock F, Dong W, Wang H, Kopf S, Kohli S, et al. Nlrp3-inflammasome activation in non-myeloid-derived cells aggravates diabetic nephropathy. Kidney Int 2015; 87:74-84.

[10] Tang SCW, Chan LYY, Leung JCK, Cheng AS, Chan $\mathrm{KW}$, Lan $\mathrm{HY}$, et al. Bradykinin and high glucose promote renal tubular inflammation. Nephrol Dial Transplant 2010; 25:698-710.

[11] de Zeeuw D, Bekker P, Henkel E, Hasslacher C, GouniBerthold I, Mehling $\mathrm{H}$, et al. The effect of CCR2 inhibitor CCX140-B on residual albuminuria in patients with type 2 diabetes and nephropathy: A randomised trial. Lancet Diabetes Endocrinol 2015; 3:687-96.

[12] Tang SCW, Lai KN. The pathogenic role of the renal proximal tubular cell in diabetic nephropathy. Nephrol Dial Transplant 2012; 27:3049-56.

[13] Nangaku M. Chronic hypoxia and tubulointerstitial injury: A final common pathway to end-stage renal failure. J Am Soc Nephrol 2006; 17:17-25.

[14] Diabetes Control and Complications Trial Research Group; Nathan DM, Genuth S, Lachin J, Cleary P, Crofford $O$, et al. The effect of intensive treatment of diabetes on the development and progression of long-term complications in insulin-dependent diabetes mellitus. N Engl J Med 1993; 329:977-86

[15] Fioretto P, Steffes MW, Sutherland DE, Goetz FC, Mauer M. Reversal of lesions of diabetic nephropathy after pancreas transplantation. N Engl J Med 1998; 339:69-75.

[16] UK Prospective Diabetes Study (UKPDS) Group. Intensive blood-glucose control with sulphonylureas or insulin compared with conventional treatment and risk of complications in patients with type 2 diabetes (UKPDS 33). Lancet 1998; 352:837-53.

[17] Wong MG, Perkovic V, Chalmers J, Woodward M, Li Q, Cooper ME, et al. Long-term benefits of intensive glucose control for preventing end-stage kidney disease: ADVANCEON. Diabetes Care 2016; 39:694-700.

[18] Tang SCW, Yiu WH. Innate immunity in diabetic kidney disease. Nat Rev Nephrol 2020; 16:206-22.

[19] Verzola D, Cappuccino L, D'Amato E, Villaggio B, Gianiorio F, Mij M, et al. Enhanced glomerular Toll-like receptor 4 expression and signaling in patients with type 2 diabetic nephropathy and microalbuminuria. Kidney Int 2014; 86:1229-43.

[20] Lin M, Yiu WH, Wu HJ, Chan LYY, Leung JCK, Au WS, et al. Toll-like receptor 4 promotes tubular inflammation in diabetic nephropathy. J Am Soc Nephrol 2012; 23:86-102.

[21] You Y-H, Quach T, Saito R, Pham J, Sharma K. Metabolomics reveals a key role for fumarate in mediating the effects of NADPH oxidase 4 in diabetic kidney disease. J Am Soc Nephrol 2016; 27:466-81.

[22] Wolf G. Renal injury due to renin-angiotensin-aldosterone system activation of the transforming growth factor-beta pathway. Kidney Int 2006; 70:1914-9.

[23] Huang XR, Chen WY, Truong LD, Lan HY. Chymase is upregulated in diabetic nephropathy: Implications for an alternative pathway of angiotensin II-mediated diabetic renal and vascular disease. J Am Soc Nephrol 2003; $14: 1738-47$.

[24] Bakris GL, Agarwal R, Anker SD, Pitt B, Ruilope LM, Rossing $\mathrm{P}$, et al. Effect of finerenone on chronic kidney disease outcomes in type 2 diabetes. N Engl J Med 2020; 383:2219-29.

[25] Heerspink HJL, Parving $\mathrm{H}-\mathrm{H}$, Andress DL, Bakris G, Correa-Rotter R, Hou F-F, et al. Atrasentan and renal events in patients with type 2 diabetes and chronic kidney disease (SONAR): A double-blind, randomised, placebo-controlled trial. Lancet 2019; 393:1937-47.

[26] Kato M, Natarajan R. Epigenetics and epigenomics in diabetic kidney disease and metabolic memory. Nat Rev Nephrol 2019; 15:327-45.

[27] Guo J, Liu Z, Gong R. Long noncoding RNA: An emerging player in diabetes and diabetic kidney disease. Clin Sci (Lond) 2019; 133:1321-39.

[28] Li B, Leung JCK, Chan LYY, Yiu WH, Tang SCW. A global perspective on the crosstalk between saturated fatty acids and Toll-like receptor 4 in the etiology of inflammation and insulin resistance. Prog Lipid Res 2020; 77:101020. 